\title{
Investigation of flow and heat transfer at the circular fins
}

\author{
Vladimir Mityakov ${ }^{1}$, Andrey Gusakov ${ }^{1}$, Vladimir Seroshtanov ${ }^{1, *}$, and Michail Grekov ${ }^{1}$ \\ Peter the Great Saint Petersburg Polytechnic University, Politekhnicheskaya St., 29, St. Petersburg, \\ Russia
}

\begin{abstract}
For simultaneous investigation of heat transfer and hydraulic parameters of finned circular tube is proposed to connect PIV (Particle Image Velocimetry) method and thermal imaging with gradient heat flux measurement. In the first series of experiments, the hollow fin was investigated. The hollow fin was heated with saturated water steam and its isothermal surface simulated the ideal (isothermal) fin. The solid fin made of titanium alloy was investigated in similar regimes for comparison. In the second series, the influence of distance between fins on the heat transfer coefficients (HTC) at the circular fin, mounted on a circular cylinder was studied. The unique Gradient Heat Flux Sensors (GHFSs) were installed at different places of the fin surface. Comprehensive method including heat flux measurement, PIV and thermal imaging allows to study flow and heat transfer at the surface of the fin in real time. Possibility of complex study of flow and heat transfer for non-isothermal fins is shown.
\end{abstract}

\section{Introduction}

A number of recuperative heat exchangers use circular tubes whose finning increase their heat transfer surface. There are a lot of finning types and techniques. However, despite wide finning application there is no universal and accurate method for calculation of finned heat exchangers. Distribution of the heat transfer coefficient (HTC) at the surface of the fin is the main problem here[1,2]. It is necessary to ensuring the exact angle between the velocity vector and tube axis [3] and space between fines. Since heat transfer and hydrodynamics are closely interrelated, it is important to study them nonselective. All of the above served as a foundation for present study.

Traditionally heat transfer and hydrodynamics have been studied during separate measurements. There are a lot of techniques and equipment for experimental research of fluid mechanics. For example PIV (Particle Image Velocimetry), hot-wire anemometry are diverse and approved. However, the investigation of heat transfer at different surfaces was troubled by the missing of heat flux sensors with the required response time. Therefore, more often, temperature was measured and HTCs were calculated from similarity equations. For more accurate comparison of flow and heat transfer, a combination of approaches that includes simultaneous measurement of heat flux per unit area and HTC using unique gradient heat

\footnotetext{
* Corresponding author: vladvik1992@gmail.com
} 
flux sensors (GHFS), velocity fields using PIV method and temperature fields visualized by thermal imaging is proposed.

\section{Investigation method}

Since the experience includes several complex technologies, it is necessary to explain the specifics of each of them separately.

\subsection{Heat flux measurement}

Heat flux per unit area was measured by the unique GHFSs with response time of $10^{-8} \ldots 10^{-9} \mathrm{~s}$. GHFSs have been developed, created and integrated into the laboratorial and industrial experience at "Thermophysics of Power Units" department of Peter the Great St. Petersburg Polytechnic University since 1996. Such a small response time of GHFS makes them practically inertialess instrument for study of most heat transfer types $[4,5,6]$. Figure 1 shows the scheme (a) and the general view (b) of the battery GHFS based on bismuth single crystals, which were used in our experiments. The choice of GHFS type was due to low surface temperatures and small temperature drop. Fig. 1, c shows three GHFSs mounted at the circular fin.

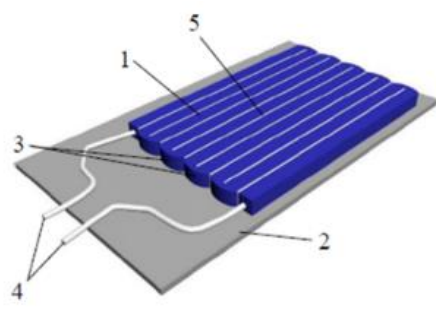

a)

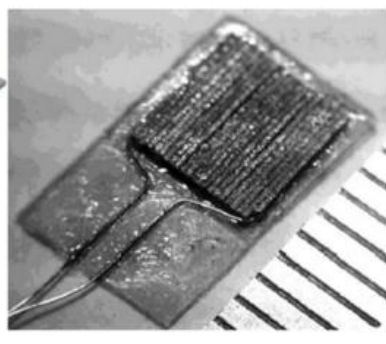

b)

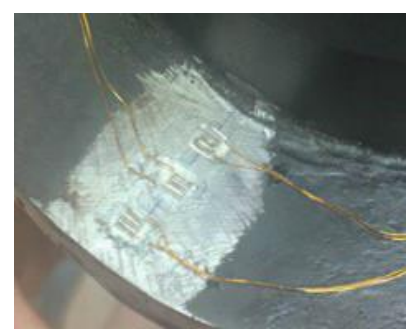

c)

Fig. 1. Scheme (a), general view (b) of the GHFS and GHFSs mounted at the fin (c). Figures denote: 1 - anisotropic bismuth strips; 2 - silica substrate; 3 - pure bismuth junctions; 4 - current outputs; 5 lavsan spacers.

The action of GHFS is based on Seebeck's transverse effect: when a heat flux passes through a plate with anisotropy of thermophysical and thermoelectric properties, thermopower arises proportionally to heat flux value. We used 5 sensors for our experiments; the dimensions of three GHFSs in plan were of $2 \times 2 \mathrm{~mm}$, the fourth sensor was of $4 \times 7$ $\mathrm{mm}$, and the fifth one was of $5 \times 5 \mathrm{~mm}$. All sensors were of $0.2 \mathrm{~mm}$ thick; their volt-watt sensitivity was about $10 \mathrm{mV} / \mathrm{W}$. GHFSs were mounted with a special compound with high thermal conductivity. Wires soldered to the GHFSs were led along the fin and the cylinder and attached to the cylinder surface at some points with a glue. Additionally, in selected range of regimes, the velocity did not exceed $15 \mathrm{~m} / \mathrm{s}$.

\subsection{PIV method}


PIV technology made it possible to visualize the flow near the surface of the fin by non-invasive method which allows to record instantaneous velocity fields in plane of the laser sheet. Principle of any PIV system operation is that tracers are fed into the air flow: fine particles (with a diameter of $2 \ldots 3 \mu \mathrm{m}$ ) which are illuminated by laser beam reshaped by lens system into the laser sheet. In our case, the supply of tracers was provided by fog machine. A digital camera fixed the image of tracers at moments of double flashes. Our POLIS system $[7,8]$ renders possible to adjust supply of tracers and the frequency of photographs under the flow regime. All photos were processed in ActualFlow program which gives the velocity field for each time step. Correlation methods of image processing to obtain particle shifts were used.

\subsection{IR imaging}

To measure temperature of the fin, we used thermographic camera FLIR P640. Software of it allows to produce powerful temperature analysis and automatic reporting. In addition, the camera has several aiming points, which allow us to measure temperature at the places where GHFSs were installed.

\subsection{Experimental model}

The first model of finned cylinder of $66 \mathrm{~mm}$ in diameter and of 600 $\mathrm{mm}$ in length was made of a steel sheet of $0.1 \mathrm{~mm}$ thick. Two circular fins with external diameter of $D=146 \mathrm{~mm}$ were mounted at the cylinder. The first fin was hollow, and it simulated "ideal" (isothermal) fin, and the second was made of titanium alloy with thermal conductivity about $k=9 \mathrm{~W} /(\mathrm{m} \times \mathrm{K})$.



a)

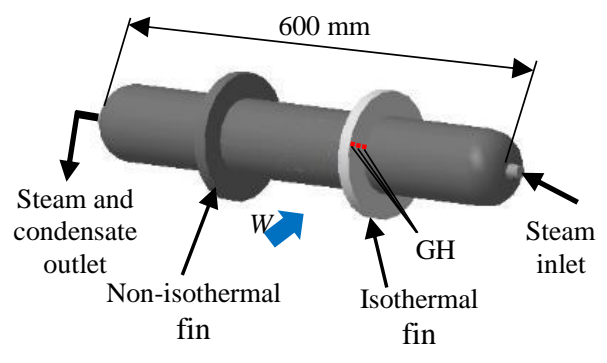

b)

Fig. 2. Scheme (a) and general view (b) of the finned cylinder model.

The second model of the finned cylinder was made similarly, but the outer diameter of the fins was of $186 \mathrm{~mm}$. The GHFSs were mounted at the fins surface (Figure 2). Both models were heated with saturated water steam under the atmospheric pressure; therefore cylinder temperature was close to $100^{\circ} \mathrm{C}$. The cylinder was turned around it's 
axis which allowed us to move the sensors in circumferential direction. For the "ideal" fin surface temperature $T_{W}=$ const for all $0 \leq \varphi \leq 180^{\circ}$ (Fig. 2, a), and for the fin made from a titanium alloy, $T_{w}$ depends on the fin height and the angular coordinate: $T_{W}=(b, \varphi)(b$ - presentposition data, $\mathrm{mm} ; 0 \leq b \leq B$ ).

The experiments were carried out in subsonic wind tunnel (Fig. 3) of "Thermophysics of Power Units" department of Peter the Great Saint Petersburg Polytechnic University.

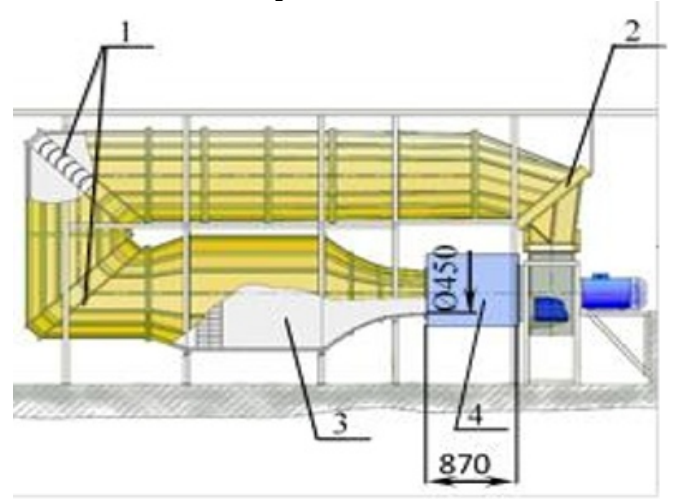

Fig. 3. Scheme of the wind tunnel: 1 - turning vanes, 2 - heat exchanger, 3 - settling chamber with honeycomb, 4 - Eiffel chamber

This wind tunnel has three features:

- Use of semiconductor-controlled rectifier (SCR's control) drive and reverser of the fan allows to produce experiments at flow speed not exceeding of $0.1 \ldots 0.2 \mathrm{~m} / \mathrm{s}$.

- The heat exchanger (cooler) connected to the cold water supply system ensures long operation of the tunnel in air, which practically does not change its temperature (the spread of the values is $\pm 0.1^{\circ} \mathrm{C}$ ).

- Eiffel camera allows to use PIV system without appreciable dispersion of tracers.

\section{Results}

Research was carried out for Reynolds number $\operatorname{Re}=\frac{W \cdot d}{v}=(0,4 \ldots .5) \times 10^{4}$ where $W-$ average air flow velocity, $\mathrm{m} / \mathrm{s} ; d$ - diameter of cylinder, $\mathrm{m} ; \mathrm{v}$ - kinematic viscosity of air, $\mathrm{m}^{2} / \mathrm{s}$.

Dependence of average (over the fin's length) HTC on Reynolds number for isothermal $(a, c)$ and non-isothermal (b, d) fins of different heights is shown at Fig. 4. Here it can be seen that HTC decreases in interval of $\varphi=120 \ldots 180^{\circ}$. This can be explained by the fact that nature of flow is such that behind the midsection of the cylinder a vortex is formed, and there is a stagnant zone near the cylinder. 


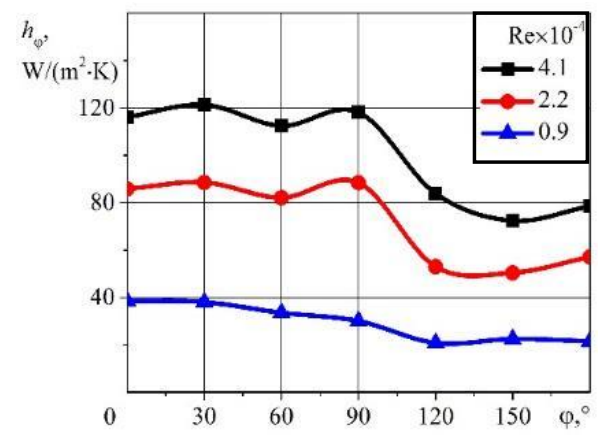

a)



c)



b)

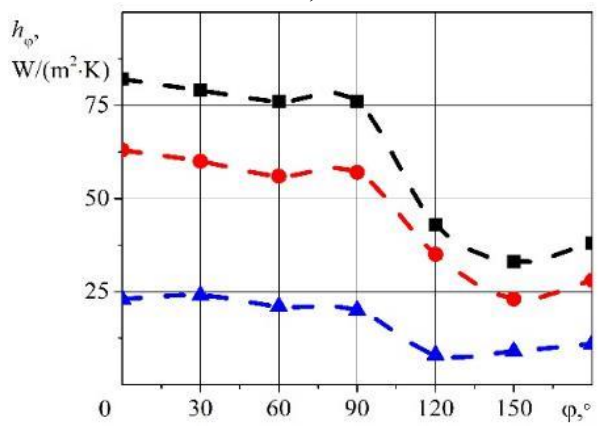

d)

Fig. 4. Dependence of average HTC on the angle of rotation $\varphi$ : a, b - for $B=20 \mathrm{~mm}, \mathrm{c}, \mathrm{d}-$ for $B=60 \mathrm{~mm}$

Local HTC was defined as:

$$
h_{\varphi}=\frac{q_{\varphi}}{T_{f}-T_{w}},\left(\mathrm{~W} /\left(\mathrm{m}^{2} \times \mathrm{K}\right)\right)
$$

$\left(q_{\varphi}\right.$-local heat flux per unit area, W/m² $T_{f}$ - air flow temperature, $\mathrm{K} ; T_{w}$ - surface temperature, $\mathrm{K}$ )

Flow temperature was measured by the multifunction tool testo-435-4 and fin surface temperature sensed by IR imaging.

Local heat flux per unit area was measured by GHFSs and equal to:

$$
q_{\varphi}=\frac{E}{S_{0} \cdot F},\left(\mathrm{~W} / \mathrm{m}^{2}\right)
$$

( $E$ - thermopower, $\mathrm{mV} ; S_{0}$ - volt-watt sensitivity, $\mathrm{mV} / \mathrm{W} ; F-$ GHFS area, $\mathrm{m}^{2}$ )

Signal E was measured with a digital voltmeter. The sensitivity was known according to predetermined calibration with measurement uncertainty of $1,3 \%$ [11].

Figure 5 illustrates velocity fields near the fins of different heights. It shows that stream pattern around the fin of $20 \mathrm{~mm}$ high is different from the flow near the fin of $60 \mathrm{~mm}$ high. 



a)
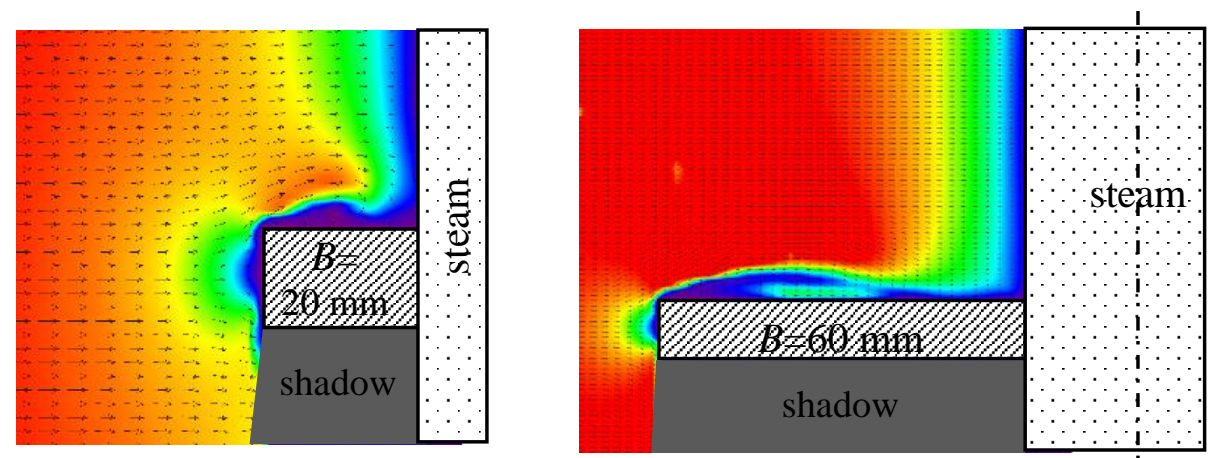

b)

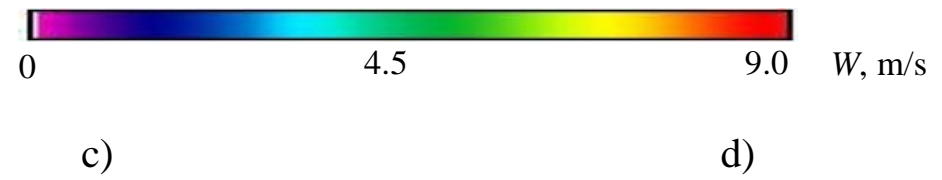

Fig. 5. Velocity fields near the fin: $\mathrm{a}, \mathrm{b}-$ for $\mathrm{Re}=2.2 \times 10^{4} ; \mathrm{c}, \mathrm{d}-$ for $\mathrm{Re}=4.1 \times 10^{4}$.

Vectors show flow direction in plane of light sheet, and colours show the speed value. Our PIV-experiments for the 60-mm-high fin show the presence of a vortex formed by flow separation. This vortex is absent at the "small" fin.

We detected additionally influence of yaw angle [12] on flow and heat transfer. Thus, in Fig. 6 time-average velocity fields near the fins, flowed at yaw angle of $\beta=5 \ldots 15^{\circ}$ are presented [13...17]. 




Fig. 6. The velocity field near the hollow fin, $\mathrm{Re}=0.4 \times 10^{4}$.

With a non-cross flow around a "small" edge, a vortex is formed and increases with increasing of yaw angle.

Also, proposed method allowed to obtain three-component fields of HTC. For example, Fig. 8 shows three-component distribution of HTC for non-cross flow. Since flow is not symmetrical, the figure shows the distributions of HTCs at the overtype (I) and undertype (II) surfaces of the fin.
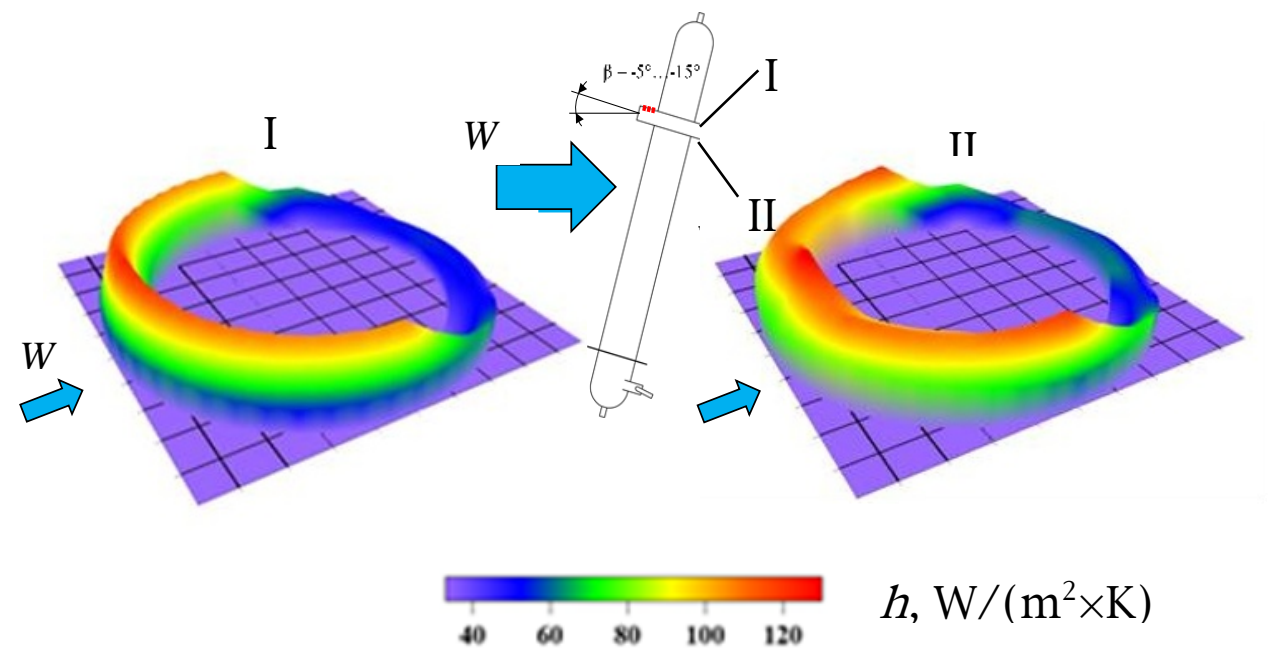

Fig. 7. Three-component distribution of HTCs.

To verify the obtained data, the heat flux at various points was measured $2 \ldots 3$ times. Our work repeats the studies $[9,10,18]$ in its methods, however, use of thermal imager makes it possible to solve the problem connected with the investigation of heat transfer at any nonisothermal surface $[19,20]$.

\section{Conclusions}

The proposed technique, including superposition of gradient heat flux measurement, PIV diagnostics and thermal imaging, allowed to study flow and heat transfer at the surface of a 
single circular cooling fin. Presence of vortices over the fin was confirmed, influence of the height of the fin on flow structure was revealed. Influence of yaw angle on flow and heat transfer was identified. It was shown that distribution of local HTC for "ideal' and real fins has a difference.

\section{References}

1. R.L. Webb, Nae-Hyun Kim. Principles of Enhanced Heat Transfer. - New York : Taylor\&Francis Group, 2005.

2. H. Hamakawa, H. Matsuoka, K. Hosokai, E. Nishida, and E. Kurihara, "Characteristics of aerodynamics sound radiated from two finned cylinders," in Proceedings of the ASME 2014 Pressure Vessels and Piping Conference, (Anaheim, California, USA), ASME, 2014.

3. E.M. Sparrow and F. Samie. Heat transfer from a yawed finned tube // Journal of Heat Transfer.-1986.-Vol. 10.-P. 479-481

4. Sapozhnikov, S. Z., Mityakov, V. Y., Mityakov, A. V., 2013, Gradient heat flux measurement fundamentals, St. Petersburg State Polytechnical University, Saint Petersburg (in Russian).

5. Korotkov, A., Loboda, V., Makarov, S., Feldhoff, A., "Modeling thermoelectric generators using the ANSYS software platform: methodology, practical applications, and prospects", Russian Microelectronics, (2017)

6. Korotkov, A, Loboda, V., Feldhoff, A., Groeneveld, D., "Simulation of thermoelectric generators and its results experimental verification", IEEE, Signals, Circuits and Systems (ISSCS) International Symposium (July 2017)

7. «POLIS» velocity profile measuring instrument [website]/ Institute of Thermophysics SB RAS, Novosibirsk - access mode: http://eng.polis-instruments.ru/

8. O. Heinz, B. Ilyushin, D. Markovich, Application of a PDF method for the statistical processing of experimental data, International Journal of Heat and Fluid Flow. 25 (5) (2004) 864-874.

9. Gusakov, A.A., Kosolapov, A.S., Markovich, D.M., Mityakov, A.V., Mityakov, V.Y., Mozhayskiy, S.A., Nebuchinov, A.S., (...), Sapozhnikov, S.Z. Simultaneous PIV and gradient heat flux measurement of a circular cylinder in cross-flow (2014) Applied Mechanics and Materials, 629, pp. 444-449.doi: 10.4028/www.scientific.net/AMM.629.444

10. Mityakov, A. Mityakov, V., Sapozhnikov, S., Gusakov, A., Bashkatov, A., Seroshtanov, V., Zainullina, E., Babich, A., "Hydrodynamics and heat transfer of yawed circular cylinder,” Int. J. Heat Mass Transf., vol. 115, pp. 333-339, (2017)

11. A.I. Pokhodun, Eksperimentalnyye metody issledovaniy. Pogreshnosti i neopredelennosti izmereniy [Experimental methods of research. Errors and uncertainties in measurements], SPbGU ITM, St. Petersburg, 2006.

12. F. Samie, E.M. Sparrow, Heat transfer from a yawed finned tube, Journal of Heat Transfer. 108 (2) (1986) 479-482.

13. E.M. Sparrow, J.M. Gorman, K.S. Friend, J.P. Abraham, Flow regime determination for finned heat exchanger surfaces with dimples/protrusions, Numerical Heat Transfer, Part A: Applications. 63(4) (2013). 245-256. 
14. Jeff.E.J. McClure, On the planar flow development and structural loading of cylinders with circular fins in cross-flow, Ph.D., University of Waterloo, Waterloo, Ontario, Canada, 2015.

15. V.M. Legkii, Ya.S. Zholudov, O.A. Gerashchenko, Local heat exchange of a single transversely round tube with external circular fins, Journal of Engineering Physics and Thermophysics. 30 (2) (1976) 178-182.

16. M. Bansal, Experimental and numerical investigation of three equispaced cylinders in cross-flow, Master's thesis, University of Waterloo, Waterloo, Ontario, Canada, 2014.

17. D. Sumner, Flow above the free end of a surface-mounted finite-height circular cylinder, A review, Journal of Fluids and Structures. 43 (2013) 41-63.

18. A. Mityakov, A. Babich, A. Bashkatov, et al., Investigating heat transfer augmentation using gradient heat flux measurement and PIV method, MATEC Web of Conferences, 2017, 3rd Siberian Thermophysical Seminar, STS 2017, 115 (10 July) (2017), No. 02006, 1-4.

19. S Z Sapozhnikov et al 2017 J. Phys.: Conf. Ser. 891012096

20. V Y Mityakov et al 2017 J. Phys.: Conf. Ser. 891012128 